Chapter 9

\title{
Management of Non-Hepatic Metastatic Disease in Colorectal Cancer
}

\author{
C. Alberto Blanco, R. Sánchez, F. Piniella, \\ D. Pescador, G. Borobio, A. García and C. Cano \\ Additional information is available at the end of the chapter
}

http://dx.doi.org/10.5772/57487

\section{Introduction}

Colorectal cancer is one of the most frequent malignant tumors and a leading cause of cancerrelated death. One third of the patients develop a metastasis during the course of the disease. Because of that, it is very important to know about the evolution of the illness, how to make a quick diagnosis and how to provide an appropriate treatment depending on the tumor and the location of the metastases.

Management of patients with colorectal metastases without the intervention of a multidisciplinary team specialized in the liver can lead to patients being denied potentially curative treatments.

\subsection{Metastatic disease}

It's necessary to have a protocol before the treatment of metastatic disease:

- Physical examination

- Laboratory test: The serum carcinoembryonic antigen (CEA) level is a valuable marker in patients with recurrent colorectal cancer. It must be remembered that about $25 \%$ of tumors do not secrete CEA.

- Imaging: Radiologic imaging is a critical component of the preoperative research on a patient with colorectal liver metastasis. In fact, it is often used to determine whether the patient should be further considered for resection.

- Surgical indications: the patient must be in acceptable health to tolerate the physiologic consequences of the surgery. Next, the primary colorectal cancer must be resected and the 
presence of other extrahepatic disease must be ruled out. Preoperative workup should include a recent colonoscopy, chest X-ray and chest CT.

- Complications: Successful outcome in hepatic surgery depends largely upon minimizing intraoperative blood loss. Excessive blood loss is not only associated with increased perioperative morbidity but also with a shorter time to recurrence and decreased survival rates after resection of colorectal liver metastases.

- Survival

\section{Non hepatic disease in colorectal cancer}

\subsection{Lung metastases}

\subsubsection{Lung metastases in CRC}

Colon cancer is a systemic disease in $19 \%$ of the patients, and the liver and lungs are the most common locations for a metastasis. Colorectal adenocarcinoma is the most common cancer leading to pulmonary metastasectomy

\subsubsection{Symptoms}

Most pulmonary metastases are asymptomatic and they are detected incidentally during the initial diagnostic staging study for a primary tumor or in the monitoring imaging studies afterwards, generally during a thoracic CT scan. Symptoms such as coughing, pain or hemoptysis mainly appear in patients with hilar involvement.

\subsubsection{Preoperative evaluation}

A solitary pulmonary nodule in a patient with a previous cancer can be bening or malignant (primary or secondary); although in most cases will be malignant (Mery et al. 2004). When there are multiple nodules, the probability of metastatic disease increases significantly.

There are no pathognomonic radiological features that distinguish the metastasis of primary tumors, even though metastasis is generally well circumscribed, spherical with smooth margins and are mainly subpleurales or periferics. However, primary lung cancers are single lesions, with irregular edges, asociated linear densities and are often more central.

Although the background and radiographic features of a lesion can provide clues as to whether an individual lesion is bening or malignant, it is not posible to distinguish reliably a metastasis from a primary lung cancer. The resection of the nodule is the most reliable method to establish the diagnosis.

The presence of pulmonary metastasis makes it necessary to carry out a complete staging. The extent of the required pulmonary resection will guide the preoperative evaluation of the patients: patients who require a pneumonectomy will need a higher level of cardiopulmonary 
reserve compared with patients who only require wedge resections (Villeneuve and Sundaresan 2009). The key for a successful surgical resection of pulmonary metastases is an adequate selection of the patient through a preoperative evaluation and a precise surgical planning. To do so, we can use the following diagnostic tests:

High-resolution helical CAT scan is a basic tool which detects approximately $20-25 \%$ more nodes that standard CT, and the detection is reliable in nodes of 2-3 mm (Remy-Jardin et al. 1993; Collie et al. 1994).

PET - An examination with Positron Emission Tomography (PET-FDG) is recommended to optimize the selection of patients who are candidates for pulmonary metastasectomy. However, we have to take into account the fact that PET has a limited sensitivity for lesions $<1 \mathrm{~cm}$ in size (Reinhardt et al. 2006). It is very useful to assess the intra-thoracic node involvement (hilar or mediastinal), local hidden recurrence of the primary tumor or in other locations such as the abdomen or the pelvis (Villeneuve and Sundaresan 2009).

The main value of PET is its high sensitivity to detect an extra-thoracic disease. In general terms, metastasectomy must not be performed unless all the deposits or foci of the disease are treatable (except when the patient is included in a specific protocol such as a clinical trial for a vaccine or symptomatic lesions that cannot be treated otherwise). In any case, a positive extrathoracic result in a PET examination is not enough evidence to rule a patient out from surgery for pulmonary metastases. All suspicious extra-thoracic FDG-highlighted areas must be biopsied before surgery.

Preoperative biopsy - In patients with highly suspicious lesions in imaging tests, the final diagnosis is often achieved after the surgical removal of the metastases. However, in many cases, a preoperative biopsy with CT-guided fine needle aspiration is a useful and less invasive method to obtain a pathological diagnosis, particularly if the diagnosis of the metastatic disease is not clear, if the patient is not a good candidate for surgery or if the patient has a primary tumor (such as testicular germ-cell cancer or lymphoma) for which surgery may not be required.

Bronchoscopy (with or without endobronchial ultrasound) is indicated as part of the evaluation in cases of lesions that are centrally located in the CT scan, patients with symptoms of involvement of the respiratory tract and some types of tumors prone to endobronchial involvement, such as breast cancer, colon cancer and renal cell carcinoma [36]. Bronchoscopy is performed before surgery, when a positive result may contraindicate an operation.

The presence of pathological mediastinal adenopathies requires a biopsy with a mediastinoscopy or ultrasound-guided endobronchial needle aspiration cytology. Most authors consider mediastinal node involvement (N2) as a contraindication for resection.

\subsubsection{Treatment}

Management of patients with pulmonary metastases, according to the guidelines of the National Comprehensive Cancer Network (NCCN) will depend on the form of presentation along time and on whether the metastases can be resected or not. Synchronous resectable 
metastases are treated with chemotherapy with or without later resection; and if they cannot be resected, then chemotherapy is indicated. Metachronous resectable metastases can be resected with or without neoadjuvant chemotherapy, and chemotherapy is indicated when they cannot be resected. The evaluation of patients in treatment with chemotherapy that can be transferred to surgery is carried out every 2 months in the selected cases (2013).

Although the quality of the currently available evidence on pulmonary metastasectomy in cases of colorectal cancer is not enough to draw conclusions on the effectiveness of this kind of surgery (Pfannschmidt et al. 2007; Fiorentino et al. 2010; Pfannschmidt et al. 2010; Salah et al. 2012; Gonzalez et al. 2013), pulmonary metastasectomy in cases of selected patients with metastatic colorectal cancer is a general practice and it is included in the clinical guidelines (Poston et al. 2011). The objective is to limit surgery only for patients who have the highest chances to benefit from it, either with longer survival rates or symptom relief, and to optimize the time of the operation.

The resectability criteria admitted by the main groups are the following (Ehrenhaft et al. 1958; Martini and McCormack 1998; Greelish and Friedberg 2000; Jaklitsch et al. 2001; Pfannschmidt et al. 2003; Kondo et al. 2005):

- Complete anatomical resection maintaining an adequate pulmonary function (McAfee et al. 1992; Regnard et al. 1998; Inoue et al. 2000; Sakamoto et al. 2001)

- Removal of the primary tumor without persistence of residual disease (R0)

- The existence of resectable extra-pulmonary metastases does not contraindicate pulmonary resection (Yano et al. 1993; Ambiru et al. 1998; Irshad et al. 2001; Rena et al. 2002); in this case, the metastases must be treatable with surgery or other therapeutic approach.

- Resectable metastases can be treated with a synchronous resection or a sequential approach.

The resection of one or more pulmonary lesions may also be indicated in a patient with a known malignant tumor when:

- A new primary lung cancer cannot be ruled out

- There are symptomatic metastases (such as bronchial obstruction with distal suppuration) that cannot be treated in any other way

- Tissue is required for a new therapeutic strategy (such as an autologous vaccine), preferably in the framework of a clinical trial.

The approach depends on the number, size, location and estability during the time in TC. In general, Video-assisted thoracoscopic surgery (VATS) is ideal for peripheral metastases single or few, stable and smaller than $3 \mathrm{~cm}$. The central lesions are likely to require a segmentectomy or lobectomy and is best addressed by open thoracotomy.

Video-assisted thoracoscopic surgery has the advantage that it is less painful, postoperative recovery is faster, hospitalization is shorter and lower long-term morbidity, especially intrathoracic recurrences (Saisho et al. 2009). Recurrence rates appear to be similar to the 
approach using thoracotomy (Saisho et al. 2009). There are no randomised trials comparing results between open resections and thoracoscopic surgery, although retrospectively patients treated by VATS have similar results to patients treated by conventional open thoracotomy (Carballo et al. 2009)

Concerning the number of metastasis, it is clear that technically the fewer there is, the better. Although there is no general consensus, ideally from technical and oncologic point of view, it would be less than five (Hellman and Weichselbaum 1995; Weichselbaum and Hellman 2011) however it is true that in the majority of cases, unique pulmonary metastasectomies are conducted and in these patients there is a greater survival advantage (Fiorentino et al. 2010). Metastases in both lungs are not a contraindication for surgery.

For some patients with metastatic CRC, repeated pulmonary metastasectomy offers an excellent opportunity for long-term survival, and it is associated with a low operative mortality rate. Patients with more than 2 metastatic nodes and a maximum diameter of the metastatic pulmonary node of more than $3 \mathrm{~cm}$ present a significantly lower survival rate (Hendriks et al. 2001; Salah et al. 2013).

In the case of patients who do not meet the criteria for a metastasectomy, there are alternative ablative techniques to locally control the lesion, such as stereotactic radiotherapy and radiofrequency ablation or cryoablation. The experience with these ablation techniques is limited, but the initial results look promising (Pennathur, Abbas et al. 2009). The role of chemotherapy is not yet defined for pulmonary metastases with a colorectal origin, because traditionally, these lesions do not show a good response to adjuvant treatments. Currently, chemotherapy has shown very good responses in primary CRC with regimens such as FOLFOX (fluorouracil, leucovorin and oxaliplatin) and FOLFIRI (fluorouracil, leucovorin and irinotecan), with or without the addition of biological agents such as bevacizumab (anti-vascular endothelial growth factor, VEGF), cetuximab and panitumumab (anti-epidermal growth factor, EGFR). These therapies have not yet been systematically studied, and they may become the most effective treatment for pulmonary metastases. As a last resort, patients whose functional state is poor are candidates for palliative treatment only (Villeneuve \& Sundaresan 2009).

External radiotherapy can be an option in very specific cases or in clinical trials, provided that the tumors are not potentially resectable.

\subsubsection{Prognostic factors}

There are many factors that have an influence on survival after a metastasectomy. The presence of one or more factors for poor prognosis does not represent an absolute contraindication for a metastasectomy (Quiros and Scott 2008). Unfavourable prognostic factors include number and size of the metastasis, inability to completely resect the entire metastatic disease, a short disease-free interval after treatment for the primary tumor and thoracic node involvement. For its part, the histology of the tumor also influences the results. 

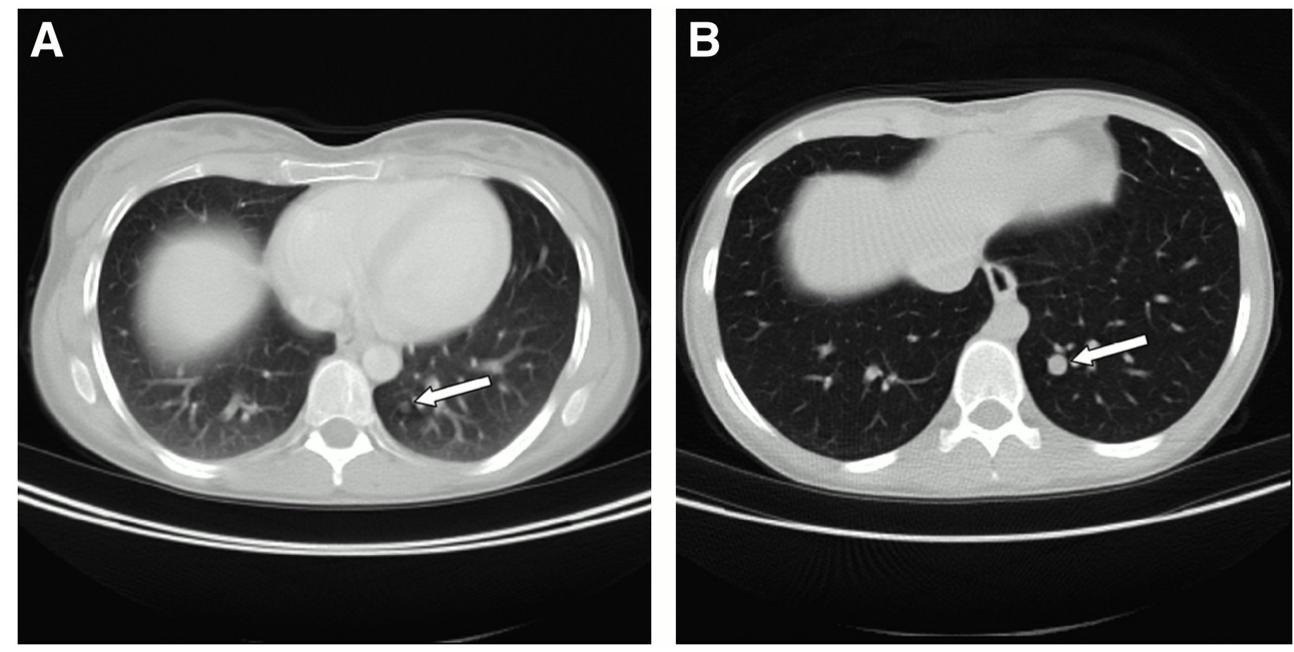

Figure 1. Comparison of chest scanning during shallow breathing $(A)$ and chest scanning with additional low-dose $C T$ during maximal inspiration (B). (A) Lung metastasis from colorectal cancer is only barely visible during shallow breathing (arrow). Also note blurred lung vessels and congested lung parenchyma in this image. (B) Metastasis can be clearly detected by low-dose CT during maximal inspiration (arrow). Additionally, lung parenchyma is well inflated, and lung vessels are displayed sharply. http://jnm.snmjournals.org/content/48/1_suppl/45S/F2.expansion.html

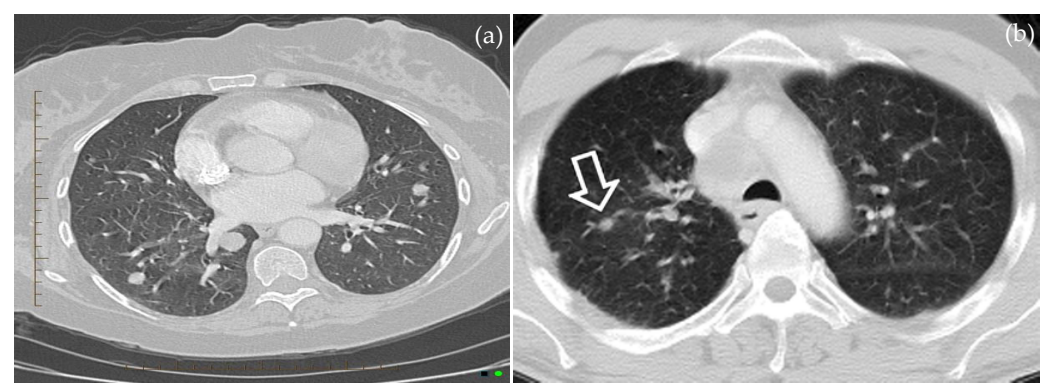

Figure 2. Multiple metastases Multiple pulmonary metastases from colorectal carcinoma. http://radiopaedia.org/articles/pulmonary-metastases, TC/PET metastases from colorectal cancer

\section{Node metastases}

\subsection{Definition and physiopathology}

Node metastases are, like with other cancers, the most important dissemination route for colorectal cancers. This makes lymph node involvement the most important factor in the prognosis and therapeutic approach for colorectal cancer. 

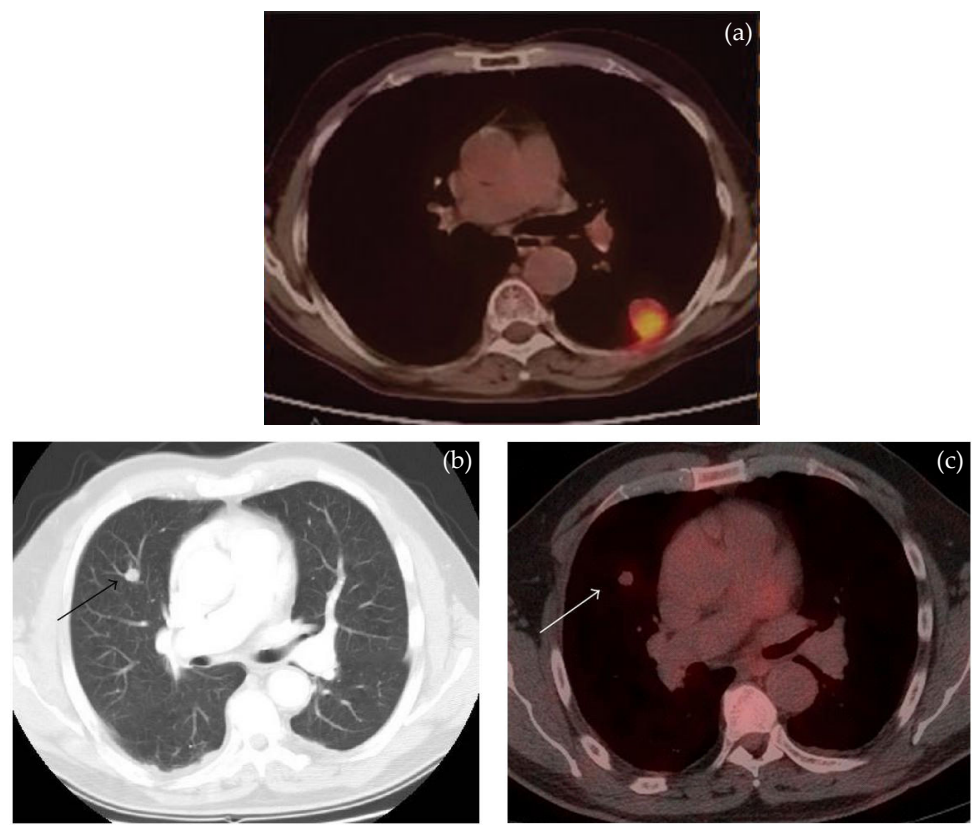

Figure 3. Patient with rectal carcinoma and mildly FDG avid metastases to lung. (a) There is a suspicious right upper lobe lung nodule (arrow) on CT in a patient with prior history of rectal carcinoma. (b) The lesion is only mildly FDG avid (arrow) on fused PET-CT. Based on these images the lesion is not definitively malignant. This may be due to the limited resolution of PET and the small lesion size or reduced cellularity. (c) Lung biopsy was subsequently performed confirming that the lesion (arrow) represented a metastatic deposit from rectal carcinoma. http://www.hindawi.com/journals/ijso/2011/846512/fig7/

The first metastases usually appear in the paracolic nodes closest to the primary tumor they are draining, and frequently only one or two nodes close to the tumor are involved. However, as the disease evolves, node propagation is larger and affects a variable amount of nodes located further away, on the arteriovenous system that depends on the intestinal segment involved. As the neoplasm moves forward, nodes from the main colic vessels that drain the intestinal neoplastic area are invaded, and the process gradually spreads upwards through the node chain that accompanies the vessels until it finally reaches the nodes that depend on the large mesenteric vessels (and, in the case of the rectum, the nodes that depend on the iliac vessels through the hemorrhoidal venous system). In short, it is known that here is a correlation between each segment of the colon and its venous and lymphatic drainage which, although there may be individual alterations, essentially depends on the superior mesenteric venous system up to the splenic flexure of the colon and, from there, up to the superior rectal vein which depends on the inferior mesenteric vein. Both are affluent to the portal vein and the structure of the lymph drainage system is essentially the same. In the area of the rectum, it has been traditionally accepted that the superior rectal venous system depends on the inferior mesenteric system, that the middle rectal system depends on the inferior mesenteric system 
and the internal iliac system, and that the inferior rectal system depends on the internal and external iliac system.

On the other hand, there are evidences that show that there are factors of the tumor cells which increase angiogenesis and lymphomagenesis, and which lead to an increased rate of node metastasis and a worse prognosis. Specifically, vascular endothelial growth factor C (VEGFC) and cyclooxygenase-2 (COX-2) (Coexpression of VEGF-C and Cox-2 in Human Colorectal Cancer and its Association With Lymph Node Metastasis Soumaoro et al. Dis Colon Rectum, March 2006 392-398).

Node involvement plays a fundamental role in the correct definition of the extent of tumor spread, together with the depth of the involvement through the wall of the colon or the rectum, the size of the tumor or the presence of metastases. The number of involved nodes and their distance to the tumor is the basis of some classic classifications, such as Dukes, Astler and Coller and the more modern TNM, whose description is already analyzed elsewhere. All decisions on the treatment must be adopted with reference to the TNM classification, instead of the old Dukes system or the modified Astler-Coller staging system (Colon and rectum. In: Edge SB, Byrd DR, Compton CC, et al., eds.: AJCC Cancer Staging Manual. 7th ed. New York, NY: Springer, 2010, pp 143-64).

Given the importance of the whole number of involved nodes in a proper staging and in order to obtain a reliable and comparable prognosis, it is necessary to establish a set of minimum quality criteria for lymphadenectomy. Otherwise, the scenario might be undervalued. Patients in stage I or II (that is, without node involvement) show five-year survival rates of $75 \%$; in contrast to survival rates with N1, which are only $45-60 \%$. Also, the higher the number of nodes studied by the pathologist (and indirectly, resected by the surgeon), the higher the staging accuracy; if that number is low, there is an undervaluing of the tumor dissemination state. (Staging accuracy in colorectal carcinoma. Wong et al. Journal of Clinical Oncology. Vol 17. no 9 1999pp2896-2900).

A panel sponsored by the American Joint Committee on Cancer (AJCC) and the National Cancer Institute of the USA and presented at the World Conference on Gastroenterology of Sydney in 1990 (J. Gastroenterol Heptol 6:325-244. 1991) has recommended that at least 12 lymph nodes be examined in patients with colon and rectal cancer in order to confirm the lack of node involvement due to the tumor. Wong et al. recommend at least the analysis of 14 nodes in pT2 and pT3 tumors.

There is a special situation with staging patients with rectal cancer and neoadjuvant chemotherapy with ypT0-2. In these cases, staging based on the surgical specimen usually shows a decrease in size and in the involvement of the wall of the intestine of the tumor, but if this is taken into account together with the reduction in the number of existing nodes in the specimen removed from the rectum, it could lead to an undervaluing with negative consequences for the patient's survival (Comparative Analisis of Limph Node Metastasis in Patients UIT ypT0-2 Rectal Cancers. Park et al. Disease of the Colon and Rectum. Volume 56: 2 (2013). Here, it is necessary to insist on the fact that, just as the primary treatment of node metastases in CRC must spread as much as possible (total mesorectal excision, or TME); in the case of patients who have undergone neoadjuvant treatment with chemotherapy it is also essential to perform a TME. 
The studies that want to ensure the diagnosis of node involvement have researched the presence of micrometastases or metastases that have been diagnosed with immunohistochemical techniques in patients in stages I and II (that is, without node involvement in microscopical terms), and they have found that the presence of micrometastases in these patients does not affect the prognosis and must not be taken into account when treating CRC (Are Lymph Node Micrometastases of any Clinical Significance in Dukes Stages A and B Colorectal Cancer? Oberg et al. Dis Colon Rectum, October 1998 ol. 41, No. 10 1244-1249).

\subsection{Sentinel lymph node}

In tumors with a metastasis that takes place through the lymphatic system (breast cancer and melanoma, mainly), there has been a great success in mapping the dissemination of the primary tumor with the so called sentinel lymph node biopsy technique. In view of this situation, several studies have started to analyze it, because lymphadenectomy is an important part in the primary treatment of CRC, both from the staging point of view and in order to prevent recurrence of the tumor if there are infiltrated lymph nodes left. Having a map of lymph dissemination similar to the one obtained with breast cancer would mean knowing the minimum required level of lymphadenectomy, or even of visceral resection. This would allow the medical team to apply an adequate surgical treatment from an oncological perspective but with the minimum morbidity and mortality. Unfortunately, it does not seem that this technique shows reliable results with colorectal cancer ("Técnica del ganglio centinela...". Sardon Ramos et al. CIR ESP. 2013 91(6) 366-371.1).

The identification of the sentinel lymph node shows a low sensitivity in the detection of metastasis and micrometastasis. Approximately one half of the adenopathies are lost if a lymphadenectomy is performed based on the results of the sentinel lymph node study. Faerden et al: sentinel node mapping in colonic cancer Disease of the colon \& rectum. Volume 51: 891-896 (2008).

\subsection{Treatment of lymphatic metastasis}

Treatment of lymphatic metastasis, given its close relation with the growth of the primary tumor, is the same as for the original lesion: primary resection with exeresis of the nodes of the lymphatic area that drain the tumor. If there is a tumor recurrence in the nodes, the patient is classified as stage IV, which means that there will be the corresponding treatment: chemotherapy and surgery and radiotherapy, which will be provisionally palliative.

\section{Bone metastases}

\subsection{Introduction}

Bone metastases from colorectal cancer are uncommon (10-23\% in autopsy cases). They usually appear late in the natural history of metastatic disease and are associated with liver or lung metastasis. Acrometastasis is reported to be $0.3-3 \%$ of all bone metastases. Cancers of the 
rectum and cecum are accompanied by bone metastasis more frequently than cancers of other portions of the colon. Signet ring cell carcinoma shows a high incidence of bone metastasis.

Pain is the most common symptom of bone metastasis. As a result of the loss of bone density affected bones become prone to fracture and injury.

Testing for bone metastasis includes X-ray and bone scanning. Open biopsy is necessary to establish the diagnosis, exclude osteomyelitis and allow treatment. Early diagnosis is important for improving quality of life in these patients.

Therapeutic management of this condition includes chemotherapy, radiotherapy and surgery, but because survival after onset of bone metastasis is very poor, palliative treatment is the main objective.

\subsection{Physiopathology}

Bone destruction secondary to metastasis is not caused by the tumor cells, but by the activation of the osteoclasts. The tumor cells secrete an osteoclast activating factor, and the osteoclasts induce the loss of cortical bone and trabecular bone. This process is divided in four stages (Mundy \& Yoneda, 1995):

1. The tumor cells adhere to the basement membrane (laminin, E-cadherin, integrins).

2. The tumor cells produce proteolytic enzymes that damage the basement membrane.

3. The tumor cells migrate via the basement membrane under the specific control of chemotactic factors.

4. The tumor cells can stimulate the activity of the osteoclasts.

Clohisy et al. have described four mechanisms that stimulate osteoclast-mediated bone destruction (Clohisy et al., 2000):

a. Stimulation of the union between the osteoclasts and the bone.

b. Stimulation of the osteoclast-mediated bone resorption.

c. Extension of the survival time of osteoclasts.

d. Acceleration of the production of osteoclasts by precursor cells.

\subsection{Clinical presentation}

a. Constitutional symptoms: Some patients report anorexia, nausea, vomiting, asthenia, malaise, and weight loss.

b. Symptoms derived from the primary location: Colorectal carcinoma usually presents itself accompanied by an alteration of the intestinal rhythm and by the expulsion of blood originated in the rectum. In advanced stages of the disease, the patient presents constipation, and a transabdominal mass can be perceived on palpation. A rectal examination needs to be performed, because tumors of the lower part of the rectum can be easily found. 
c. Symptoms derived from the metastatic disease: Regardless of the symptoms that metastases may produce on other regions, bone metastases can lead to:

a. PAIN in the affected area, or referred pain, which may be of insidious, and either progressive or sudden onset, and it may be slight and intermittent or continuous and activity-related. Night pain is a typical symptom, and it does not always disappear with oral analgesics, unlike the pain that derives from degenerative processes, such as osteoarthritis, which increases with loads and articular mobility. When pain affects a long bone, it is easily located by the patient, but when it affects the pelvis or the spinal column, the pain makes it difficult to properly locate the lesion. When it affects the femur or the tibia (load-bearing bones), patients report pain when walking, although the pain usually appears when the bone destruction levels are over $50 \%$ and they indicate an imminent fracture.

b. SWELLING: It may be a sign of lesion aggressiveness when the tumor invades the cortical bone and affects soft tissue. This presentation is characteristic from colorectal carcinoma, renal carcinoma and melanoma.

c. FUNCTIONAL DEFICIT: it appears as a consequence of pain. It may be a result of a medullary or radicular involvement in the case of spinal metastases.

d. IMMINENT FRACTURE: It is a fracture that can appear as a result of a physiological load. Anamnesis and plain X-ray are necessary for the diagnosis, and the cortical involvement, the location and characteristics of the lesion (lytic, sclerotic or mixed) and the existence of fracture lines must be assessed. Permeating and lytic lesions of the proximal third of the femur are prone to fractures. Pain after radiation is also a sign of an imminent fracture. In cases in which an imminent fracture is expected on an active patient, a prophylactic fixation is recommended, especially in load-bearing bones.

\subsection{Diagnostic assessment}

In the context of colorectal carcinoma, bone metastases normally appear when the disease is already in an advanced stage (with metastases on other areas), and when the diagnosis has already been established. For this reason, a histological diagnosis is not usually necessary, and a treatment can be planned in advance. However, we must also take into account the fact that in $1-2 \%$ of the cases, the osteolytic lesion is unrelated to the primary tumor, which means that a biopsy is advisable.

\subsubsection{Complete physical examination}

Including the thyroid gland, breasts, lungs and digestive system.

\subsubsection{Laboratory analyses}

1. COMPLETE BLOOD COUNT: Anemia, leukopenia or thrombocytopenia may be a sign of medullary involvement.

2. ESR: High levels may indicate a myeloma or an active process. 
3. ELECTROPHORESIS OF SERUM PROTEINS: They can show a monoclonal gammopathy and they can confirm a possible myeloma diagnosis.

4. BIOCHEMICAL ANALYSIS: It can rule out hyperparathyroidism.

5. ALKALINE PHOSPHATASE: It shows high levels in cases of advanced metastatic disease. Very high levels show an unfavourable prognostic factor.

6. CARCINOEMBRYONIC ANTIGEN: Its levels are high in digestive or hepatocellular carcinomas.

7. PROSTATE-SPECIFIC ANTIGEN: It can detect a prostate carcinoma.

8. HEPATIC ENZYMES AND SERUM ELECTROLYTES: They can show bone and liver involvement.

\subsubsection{Imaging tests}

1. ANTEROPOSTERIOR AND LATERAL X-RAYS OF THE LESION: In order to assess an imminent fracture and to analyse the information they provide.

2. THORACIC X-RAY: In order to see the existence of carcinoma or lung metastases.

3. THORACIC AND ABDOMINAL CT SCAN: In order to assess the existence of possible visceral metastases.

4. Tc99m BONE SCINTIGRAPHY: In order to assess bone lesions.

Data from the clinical record, an exhaustive physical examination, blood tests and imaging tests identify more than $85 \%$ of all the primary tumors that appear as a bone metastasis. The following tests could also be performed, albeit only when required:

- NMR: It is seldom recommended in cases of isolated bone lesions (fig. 1), but it may be useful in cases of a single metastasis in which a resection can be performed, in order to rule out skip metastases or metastases inside the bone and on the vertebrae, due to its excellent properties for the exploration of the bone marrow.

- POSITRON EMISSION TOMOGRAPHY (PET): This imaging technique is becoming more and more important in the field of orthopaedic oncology. It uses [18F]2-fluoro-2-deoxyDglucose (FDG) as a tracer. This is a glucose analog which is taken to the cells by a group of proteins. This marker is absorbed by malignant tissue with an increased metabolic activity. PET scans have a very high sensitivity, and it is an important technique for the identification of primary lesions and other metastases. It can establish the difference between a local recurrence and a scar, and it is also useful in the assessment of response to treatment.

\subsubsection{Biopsy}

Puncture biopsy is an excellent way to confirm a diagnosis of bone metastasis. CT-guided fineneedle aspiration and thick- or trephine-needle biopsies are very precise techniques, and they 
are easy to use. The orthopaedic surgeon must choose the exact location, taking into account the location of the lesion, viable access routes and, whenever possible, the final incision line of the operation, in case of resection surgery, excising the entire area of the biopsy, because it might be contaminated.

When finding certain locations (usually on the pelvis), a CT scan may be necessary in order to identify the best point and route of access that will reach the metastatic area and to avoid regions with reactive sclerotic bone, because these parts may not have tumor cells.

If colorectal carcinoma presents itself with a bone metastasis and the lesion is biopsied, the biopsy may not always provide a diagnosis for the primary tumor, because a tissue compatible with adenocarcinoma does not always tell the difference between primary tumors of the digestive system, prostate, breast and lung.

\subsection{Supportive measures}

\subsubsection{Analgesic therapy}

Around $70 \%$ of all patients with a bone metastasis report pain at some point along the course of the disease. The physiopathological pain may be due to medullary compression, distension of the periosteum or peripheral neurovascular involvement, as well as to pathological fractures, whenever they are present and mediated by substances such as histamine, substance P or other cytokines.

\subsubsection{Bisphosphonates}

Metastatic osteolysis is caused by the stimulation of osteoclast activity. For this reason, bisphosphonates can play an important role in this process, because they inhibit the osteoclast activity. They bind with the mineral bone matrix and they have a great physicochemical impact on the hydroxyapatite crystals.

Some authors have suggested that they are not only useful in the treatment of pain and the prevention of osteolytic complications, but that they can also modify the natural course of evolution of cancer in some cases, due to the effect they have on some intermediate products, such as growth factors.

Ross et al. carried out a systematic review of all randomized essays on patients with bone metastasis. It is a meta-analysis based on 18 randomized studies in which different bisphosphonates have been compared with a placebo or between themselves. Most of these studies were performed on patients with breast carcinoma (Ross et al, 2004). The review showed a decrease in the incidence and an increase in the time until the appearance of bone complications, with a better evolution of pain and functional capacity, with regard to the control group who received a placebo. Treatment with oral bisphosphonates (clodronate, etidronate) caused a decrease in the number of spinal and non-spinal fractures, but it had no effect on the indications of radiotherapy or in hypercalcaemia. 


\subsubsection{Treatment of hypercalcaemia}

Hypercalcaemia affects $10-40 \%$ of cancer patients at some point, and it causes anorexia, nausea, vomiting, polydipsia, polyuria, dehydration, constipation, confusion and coma.

It is the result of PTHrP production, which activates bone metabolism and induces an excess of osteoclast activity. Osteoclasts are then stimulated by local factors produced by tumor cells, such as interleukin 6. Moreover, calcium levels are also increased due to lower levels of renal calcium elimination, because PTHrP acts on the renal receptors of the parathyroid hormone and it increases calcium resorption on the renal tubule. Polyuria and reduction of intravascular volume appear as a consequence, and for this reason, the initial treatment with these patients is rehydration with intravenous saline serum in order to balance the intravascular volume and to improve glomerular filtration and renal secretion of calcium.

Calcitonin inhibits osteoclasts and it has a rapid effect, although for a brief period of time. For this reason, it is mainly used in emergency treatments.

Plicamycin normalizes calcium levels in up to $50 \%$ of the cases, but its serious adverse effects make it unadvisable to use it.

\subsection{Non-surgical treatment}

\subsubsection{Treatment of metastatic bone disease secondary to colorectal carcinoma}

The treatment of bone metastases derived from colorectal tumors is the same as the treatment for other metastases caused by other tumors. Surgical resection of the primary tumor, together with chemotherapy and radiotherapy for the rectal cancer is the treatment of choice, depending on the cases.

\subsubsection{Radiotherapy}

Radiotherapy is the most widely used palliative treatment for bone metastasis. It is the treatment of choice for painful lytic bone metastases without short-term risk of fracture, and it is combined with surgery when there is an imminent fracture or when the fracture has already taken place. It leads to the necrosis of tumor cells, which makes it possible for the bone tissue to regenerate afterwards. The result is pain relief and, later on, a re-calcification of the destroyed areas of the bone, which is important for the functional recovery of the patient and the prevention of pathological fractures.

\subsubsection{Surgical treatment}

Surgery for bone metastases requires a previous complete general and local assessment. It presents its own indications, objectives, techniques and means, and it is associated to a program for postoperative radiotherapy that follows the lines that have been previously described. 


\section{Brain metastases}

The increasing incidence of brain metastases in patients with metastatic colorectal cancer has been attributed to the longer survival rates seen with newer systemic therapies. Compared to the era when 5-fluorouracil was the primary agent for metastatic disease, median survival has increased markedly with the introduction of oxaliplatin, irinotecan, and biologic therapies (from 6 to 7 to approximately 24 months).

However, the incidence of brain metastases in metastatic colorectal cancer is still low, 2.3 percent in one of the series. Brain metastases are usually a late-stage phenomenon, and the vast majority of patients have metastases in other sites, particularly the lung. Outcomes are poor, despite aggressive treatment.

The most common mechanism of metastasis to the brain is by hematogenous spread. Metastases are usually located directly at the junction of the gray matter and white matter where blood vessels decrease in diameter and act as a trap for clumps of tumor cells. Brain metastases also tend to be more common at the terminal "watershed areas" of arterial circulation. The distribution of metastases roughly follows the relative weight and blood flow in each area:

- Cerebral hemispheres - approximately 80 percent

- Cerebellum - 15 percent

- Brainstem -5 percent

Different primary tumors may have a predilection for metastasis to different areas within the brain. Gastrointestinal tumors more commonly metastasize to the posterior fossa.

\subsection{Clinical presentation}

Brain metastases have highly variable clinical features and should be suspected in any cancer patient who develops neurologic symptoms or behavioural abnormalities. However, multiple other causes can also be responsible. In the majority of patients, a gradually expanding tumor mass and its associated edema cause symptoms. Less commonly, intratumoral hemorrhage, obstructive hydrocephalus, or embolization by tumor cells result in symptoms.

Headache: Headaches occur in approximately 40 to 50 percent of patients with brain metastases. The frequency is higher when multiple lesions are present or a metastasis is located in the posterior fossa.

Focal neurologic dysfunction: Focal neurologic dysfunction is the presenting symptom of 20 to 40 percent of patients. Hemiparesis is the most common complaint but the manifestations depend upon the location of the metastases.

Cognitive dysfunction: Cognitive dysfunction, including memory problems and mood or personality changes, is the presenting problem in 30 to 35 percent of patients. 
Seizures: New onset of seizures is the presenting symptom in 10 to 20 percent of patients. Seizures in patients with brain metastases are almost exclusively associated with supratentorial disease.

Stroke: Another 5 to 10 percent present acutely due to stroke caused by hemorrhage into a metastasis, hypercoagulability, invasion or compression of an artery by tumor, or embolization of tumor cells.

\subsection{Diagnosis}

Imaging studies provide useful information but brain biopsy is necessary in some cases for a definitive diagnosis.

Contrast-enhanced MRI is the preferred imaging study for the diagnosis of brain metastases.

Biopsy should be performed when the diagnosis of brain metastases is in doubt. This is particularly important in patients with a single lesion.

Positron emission tomography (PET) may also be useful in these patients by finding other sites of metastatic disease.

\subsection{Treatment}

Patients with BM from CRC have a poor prognosis, because they often have substantial extracranial metastatic disease.

Traditionally, the therapeutic goal in many of these patients has been to palliate debilitating neurologic symptoms, because most of these patients die of systemic disease. However, new advances in metastatic CRC management-including the incorporation of monoclonal antibody therapies bevacizumab, cetuximab, and panitumumab - are enhancing the outcomes of patients with systemic disease.

\section{Ovarian metastases}

Isolated ovarian metastases from primary CRC occur with a low frequency. The incidence of ovarian metastases (synchronous or metachronous) in patients with CRC is 1 to 14 percent. Bulky ovarian metastases are often symptomatic and less responsive to systemic chemotherapy than are other sites of disease. Resection is associated with fairly low morbidity and, in some cases, may improve quality of life and prolong survival, even in the setting of widespread extraovarian metastatic disease. There is a debate regarding prophylactic oophorectomy at the time of curative resection for primary CRC.

There is a rare variety of ovarian metastasis known as Krukenberg tumor (KT), which has been the focus of extensive research due to its poor prognosis. Although the age range of patients is highly variable, KT usually appears in premenopausal women, and for some authors, the diagnosis of primary tumor after KT is a factor for poor prognosis. They are usually large 
bilateral lesions, solid and bulbous, and they have a gastrointestinal origin in more than $90 \%$ of the cases. Although the form of presentation and the treatment are not different from the rest of metastatic ovarian lesions secondary to a primary tumor with a digestive origin, the prognosis is worse. It has been proven that a combined therapy of radical surgery and radiochemotherapy can achieve a slight improvement in long-term survival rates.

\section{Peritoneum metastases}

\subsection{Definition and physiopathology}

Colorectal cancer is a pathological process that spreads through the lymphatic channels through hematogenous ways and through the invasion of the intestine wall. These mechanisms result in metastases to the lymph nodes, liver and also peritoneal dissemination. Although the spread to the lymph and venous systems implies the existence of a local invasive process, peritoneal dissemination can appear in tumors with high as well as low malignancy. The dissemination of the cancer that leads to liver and lymph node metastases takes place before the surgical resection of the primary colorectal neoplasm. Peritoneal dissemination, as well as dissemination in the resection area (local recurrence) can also take place as a result of the surgical trauma associated to the resection of the primary neoplasm. The filtration of malignant cells through the severed lymph channels can also be a mechanism in this intraoperative phenomenon of cancer dissemination. Dissemination inside the peritoneal cavity is one of the most severe forms of carcinomatous dissemination from the colon, because it rapidly takes away - provisionally - all hope for a surgical resection of the lesion. Together with lymphatic and hematogenous dissemination, transcoelomic spread is one of the routes of tumor dissemination. In the early stages of peritoneal invasion, transcoelomic spread may be limited to the neighbouring structures just around the primary tumor, with a potential development of isolated carcinoid plaques in the adjacent peritoneum. It is of course a possibility that some of these nodes are not originated in a transperitoneal spread but in a dissemination of the subperitoneal lymph nodes, as Miles proposes (1926). In a later stage, peritoneal metastases spread until there is a diffuse peritoneal carcinomatosis in which there are tumor nodes that spread through the entire parietal peritoneum, greater omentum and adjacent viscera, and abundant ascites appears (Cirugía del ano, recto y colon. John Golligher 2ª edición). For Hara (Hara et al. Comparative analysis of intraperitoneal minimal free cancer cells between colorectal and gastric cancer patients using quantitative RT-PCR: possible reason for rare peritoneal recurrence in colorectal cancer. Clin Exp Metastasis 2007;24:179-89), peritoneal metastases take place in two stages: first of all, the tumor cells detach themselves from the serosal surface of the primary tumor and are transported in the peritoneal cavity, and in the second stage, malignant cells in the peritoneum adhere to places like the omentum and the mesenterium, and they grow and spread through the peritoneal cavity afterwards.

Four characteristics have been identified as risk factors for tumor cell exfoliation in the peritoneal cavity: 1. Depth of the invasion, 2. Involvement of lymph nodes, 3. Lymph node 
invasion, and 4 . Venous invasion (Peritoneal cytology in colorectal cancer. Noura et al: Diseases of the Colon \& Rectum. volume 52: 7 2009).

The identification of these tumor cells with preoperative peritoneal washing cytology can identify those patients at risk of presenting a peritoneal recurrence of the tumor process.

Peritoneal carcinomatosis is a common form of recurrence, and it is frequently the only one, after surgical curative treatment in digestive and gynecological tumors. It is logical to think that the dissemination of tumor cells by the tumor is a common previous mechanism for peritoneal carcinomatosis of abdominal tumors.

There are studies about the mechanisms and incidence of intraperitoneal dissemination of CRC that have contributed to a better comprehension and a different perception of the pathological basis of peritoneal carcinomatosis. Although the incidence rates for the presence of tumor cells during the resection of primary tumors widely vary, the presence of free tumor cells in the peritoneal cavity (similar to the micrometastases in the blood or the bone marrow) is not an independent prognostic factor, and it seems possible that these tumor cells can effectively contribute to the failure of the treatment at an intraperitoneal level. In fact, two studies found a correlation between the presence of free tumor cells in the peritoneal cavity and the recurrence of the tumor in the peritoneal cavity (Peritoneal Carcinomatosis of Colorectal Origin. M.J. Koppe. Ann Surg 2006 Febrero. 243(“): 212-222).

Approximately $50 \%$ of the patients with CRC stage IV present peritoneal carcinomatosis, and approximately $25 \%$ of the patients present a recurrence of their tumor in the peritoneal cavity (peritoneal carcinomatosis), without a clear involvement of the liver or the lungs (Chu DZ, Lang NP, Thompson C, et al. Peritoneal carcinomatosis in nongynecologic malignancy: a prospective study of prognostic factors. Cancer. 1989;63:364-367. Sugarbaker PH, Cunliffe WJ, Belliveau J, et al. Rationale for integrating early postoperative intraperitoneal chemotherapy into the surgical treatment of gastrointestinal cancer. Semin Oncol. 1989;16:83-97).

Local or peritoneal metastases are factors that represent a poor prognosis for colorectal cancer (Graf et al, 1991; Mahteme et al, 1996; Shepherd et al, 1997; Assersohn et al, 1999) and their treatment is still an important challenge.

The EVOCAPE study shows an average survival of 5.2 months in patients with peritoneal carcinomatosis of colorectal origin. (Sadeghi B, Arvieux C, Glehen O, et al. Peritoneal carcinomatosis from non-gynecologic malignancies: results of the EVOCAPE Multicentric prospective study. Cancer. 2000;88:358-363.).

Patients with peritoneal metastases or locally advanced tumors without distance metastases can benefit from cytoreductive surgery with intraperitoneal chemotherapy (Mahteme et al. British Journal of Cancer (2004) 90 403-407).

The average survival rate for patients with carcinomatosis treated with chemotherapy is around 6-12 months (Gramont er al.2000), although this does not factor the modern therapeutic approaches that include immunomodulating agents, which have not yet been evaluated enough and appeared after the year 2000. 


\subsection{Peritoneal cytology}

In this situation, peritoneal cytology before the resection of the primary tumor and, when needed, cytoreduction, is a useful tool to assess the prognosis of the patients (both with and without peritoneal carcinomatosis). And it can also be useful when deciding whether to choose systemic or intraperitoneal chemotherapy.

In a multivariate analysis, peritoneal cytology appears as an independent predictor for survival in patients with tumors pT3 or pT4 (Peritoneal cytology in colorectal cancer. Noura et al: Diseases of the Colon \& Rectum. volume 52: 7 2009). Patients with negative cytology results show better five-year survival rates in cases in which at least the serosal layer is affected by the tumor than patients with positive results (68\% vs. $20.6 \%$ ). These results are also reflected in the fact that patients with positive cytology results present a higher rate of peritoneal dissemination than patients with negative cytology results. Some patients with macroscopic peritoneal dissemination and negative cytology have been proven to have survived for a long time (more than 10 years, in some cases); whereas patients with peritoneal dissemination and positive cytology results show five-year survival rates close to zero. When the peritoneal cytology results are positive, monitoring has to be particularly careful in order to obtain an early detection of recurrence (Peritoneal Cytology in Colorectal Cancer. Nishikawa et al. Disease of the Colon and Rectum. Volume 52:12 (2009). All these promising results with regard to peritoneal cytology must be included in the evaluation, prognosis and therapeutic approach, and this requires subsequent prospective studies that homogenize the way in which the cytology is performed (technique, staining, etc.) and its inclusion in the different studies, such as its inclusion in the selection protocols to perform Sugarbaker technique.

\subsection{Treatment}

Peritoneal carcinomatosis and ascites are usually signs of advanced colorectal cancer, and survival rates, as we have said, are low. However, a more aggressive approach for surgical cytoreduction of the peritoneal disease has been used as in the treatment for ovarian cancer, primary peritoneal cancer and appendicular mucinous tumors such as pseudomyxoma and cystadenocarcinoma, and it shows better results than palliative surgery and conventional chemotherapy by themselves. The extrapolation of these therapies to the treatment of CRC seems inappropriate, because these tumors are biologically different, and they are mainly of a low grade. The arrival of intraperitoneal chemotherapy combined with peritoneal debulking seems to offer an increased survival rate in selected patients with colorectal carcinomatosis. Sugarbaker described in 1995 the surgical techniques that lead, when this is not possible, to a complete resection of the peritoneal neoplastic disease. As in other scenarios of resectable metastatic disease, it is advisable to consolidate the results obtained thanks to surgery with a complementary treatment that eradicates the residual microscopic disease. The intraperitoneal administration of certain cytostatic drugs leads to a higher exposure of the peritoneal surface to the drugs than with the usual systemic administration. The perioperative administration (hypertermia-modulated intraoperative administration and/or early postoperative administration) avoids the difficulties that have been traditionally associated with the intraperitoneal 
administration of cytostatic drugs (difficulty of access, erratic distribution, pain...), which have been one of the main causes of its poor reception by specialists in medical oncology.

This way, and after decades of preclinical and clinical efforts, cytoreductive surgery combined with preoperative hyperthermic intraperitoneal chemotherapy (HIPEC) appear in this new century as an indissoluble and feasible multimodal strategy with proven effectiveness in the treatment of selected cases of peritoneal carcinomatosis in colorectal cancer, although, as we have said before, it can also be useful for other tumors (González-Moreno S. Cirugía citoreductora y quimioterapia intraperitoneal perioperatoria para las neoplasias con diseminación peritoneal: ha llegado el momento. Cir Esp. 2005;78(6):341-3)(Sugarbaker PH, Mora JT, Carmignani P, Stuart OA, Yoo D. Update on chemotherapeutic agents utilized for perioperative intraperitonealchemotherapy. Oncologist. 2005;10:112-22.)(Treatments and Outcomes of Peritoneal Surface Tumors Through a Centralized National Service (United Kingdom) S. Rout, Diseases Of The Colon \& Rectum Volume 52: 10 (2009).

There are protocols for the selection of patients which, among other things, try to establish the mass of the tumor and its location by mapping the abdominal cavity in order to assess the size of the tumor and the possibility of achieving an adequate cytoreduction. Laparoscopy is a technique that is currently included in the treatment protocols when there is suspicion or preoperative evidence of peritoneal carcinomatosis. After obtaining a laparoscopic diagnosis of the affected areas (including images obtained during the process, if possible), the patient is sent to a health centre with experience in cytoreductive surgery and HIPEC in order to assess the procedure (commonly referred to as Sugarbaker technique).

The prognosis and results depend on the level of cytoreduction. It is necessary to take into account the fact that this cytoreduction goes through a peritonectomy and the resection of visceral metastases that sometimes involve several days of surgery, with the corresponding increase of morbidity and mortality. For this reason, it is essential to have specialized or experienced centres when assessing the results.

As we have said, the level of cytoreduction is directly related to the prognosis. The success rate of cytoreduction has been established according to different systems, although it is generally classified as CCR 0 (Completeness of Cancer Resection) when there is no microscopic tumor, CCR 1 when there are no nodes larger than $0.5 \mathrm{~cm}$ and CCR 2 when there are clearly visible tumors (more than $0.5 \mathrm{~cm}$ ). Average survival is 33 months for CCR 0, 12.5 months for CCR 1 and 8.5 months for CCR 2 (Glehen O, Cotte E, Schreiber V, Sayag-Beaujard AC, Vignal J, Gilly FN. Intraperitoneal chemohyperthermia and attempted cytoreductive surgery in patients with peritoneal carcinomatosis of colorectal origin. Br J Surg. 2004;91:747-754).

The most commonly used chemotherapeutic agents are mitomycin $\mathrm{C}$ and cisplatin combined with 5-FU. These agents are heated to $47-59^{\circ} \mathrm{C}$ and inserted in the peritoneal cavity, which lets the surgeon spread it to all necessary spaces. They are left in the cavity for one hour and a half to two hours, and then they are drained. The catheters are left in place for postoperative drainage (Royal RE, Pingpank JF Jr. Diagnosis and management of peritoneal carcinomatosis arising from adenocarcinoma of the colon and rectum. Semin Oncol. 2008;35:183-191). 
Morbidity rates in the procedure of cytoreduction and HIPEC are around 30\% and $60 \%$, and mortality is less than $2 \%$. Logically, covering the logistic needs for intensive care, chemotherapy and trained surgeons with an established protocol allow for an adequate morbidity and mortality. This therapy must be applied to patients in whom a complete or almost complete cytoreduction can be achieved. With these conditions, the existing studies (although retrospective) show an important improvement of survival, with some results showing a five-year survival rate of $49 \%$ when cytoreduction is complete. (Yan TD, Black D, Savady R, Sugarbaker $\mathrm{PH}$. Systematic review on the efficacy of cytoreductive surgery combined with perioperative intraperitoneal chemotherapy for peritoneal carcinomatosis from colorectal carcinoma. J Clin Oncol. 2006;24: 4011-409).

It is possible to apply delayed and repeated intraperitoneal systemic chemotherapy after the cytoreduction with acceptable morbidity rates. It is also possible to administer delayed intraperitoneal chemotherapy in up to $83 \%$ of the patients who had previously undergone cytoreduction (Delayed Repeated Intraperotoneal Chemotherapy. Fajardo et al. Diseases of Colon and Rectum. Volumen 55.: 10 (2012)).

On the other hand, there are reasons to be optimistic about the use of immunomodulating treatments (targeted monoclonal antibodies) which, when combined with other 'traditional' chemotherapeutic approaches, are showing some really promising results. We still need more studies that let us know what conditions lead to a successful outcome (such as the KRAS status of the primary tumor) and also whether they have a beneficial effect in peritoneal carcinomatosis.

Chemotherapy and targeted therapies: There are currently eight active and approved drugs for patients with metastatic colorectal cancer which are used separately or combined with other drugs:

5-FU, Capecitabine, Irinotecan., Oxaliplatin, Bevacizumab, Cetuximab, Aflibercept and Panitumumab.

These chemotherapeutic agents, some of which are monoclonal antibodies, can be combined in different ways and represent an encouraging future for patients with peritoneal metastases who were previously called 'terminally ill'.

\subsection{Treatment of metastatic disease}

Initial management of the primary site in patients who present with stage IV disease is controversial, and there are no data from prospective randomized studies to guide treatment. In general, the choice and sequence of treatment is guided by the presence or absence of symptoms from the primary tumor and whether or not the metastases are potentially resectable.

Surgery provides a potentially curative option for selected patients who present with limited metastatic colorectal cancer.

Management in patients with unresectable metastatic disease: systemic and hepatic arterial infusion chemotherapy may be useful treatment options in patients with unresectable disease.

Other established treatment is radiofrequency ablation. 


\section{Author details}

C. Alberto Blanco ${ }^{1 *}$, R. Sánchez ${ }^{2}$, F. Piniella ${ }^{3}$, D. Pescador ${ }^{3}$, G. $_{\text {Borobio }}{ }^{3}$, A. García $^{3}$ and

C. $\mathrm{Cano}^{3}$

*Address all correspondence to: titoberto2002@hotmail.com

1 Hospital Santos Reyes, Aranda de Duero (Burgos), Spain

2 Health Care Centers of Aranda de Duero (Burgos), Ávila, Spain

3 Health Care Centers of Aranda de Duero (Burgos), Salamanca, Spain

\section{References}

[1] Hara et al. Comparative analysis of intraperitoneal minimal free cancer cells between colorectal and gastric cancer patients using quantitative RT-PCR: possible reason for rare peritoneal recurrence in colorectal cancer. Clin Exp Metastasis 2007;24:179-89.

[2] Noura et al. Peritoneal cytology in colorectal cancer.: Dis Colon Rectum. Vol 52: 7 (2009)

[3] Koppe et al. Peritoneal carcinomatosis of colorectal origin.. Ann Surg 2006 243: 212-222.

[4] Chu et al. Peritoneal carcinomatosis in nongynecologic malignancy: a prospective study of prognostic factors. Cancer. 1989;63:364-367.

[5] Sugarbaker et al. Rationale for integrating early postoperative intraperitoneal chemotherapy into the surgical treatment of gastrointestinal cancer. Semin Oncol. 1989;16:83-97.

[6] Sadeghi et al. Peritoneal carcinomatosis from non-gynecologic malignancies: results of the EVOCAPE Multicentric prospective study. Cancer. 2000;88: 358-363.

[7] Wagner et al. Eartly postoperative intraperitoneal chemotherapy folloeonng cytoreductive surgery for appendiceal mucinous neoplasms with isolated peritoneal metastasis. Dis Colon Rectum Vol 55: 4 (2012) 407-415.

[8] Mahteme et al. Improve survival in patients with peritoneal metastases from colorectal cancer: a preliminary study. British Journal of Cancer (2004) 90 403-407.

[9] Nishikawa et al. Peritoneal Cytology in Colorectal Cancer.. Dis Colon Rectum. Vol $52: 12(2009)$ 
[10] González-Moreno S. Cirugía citoreductora y quimioterapia intraperitoneal perioperatoria para las neoplasias con diseminación peritoneal: ha llegado el momento. Cir Esp. $2005 ; 78(6): 341-343$

[11] Sugarbaker et al.. Update on chemotherapeutic agents utilized for perioperative intraperitonealchemotherapy. Oncologist. 2005;10:112-22

[12] Rout et al. Treatments and Outcomes of Peritoneal Surface Tumors Through a Centralized National Service (United Kingdom) Dis Colon Rectum Volume 52: 10 (2009)1705-1715.

[13] Glehen et al. Intraperitoneal chemohyperthermia and attempted cytoreductive surgery in patients with peritoneal carcinomatosis of colorectal origin. Br J Surg. 2004;91:747-754.

[14] Royal et al.. Diagnosis and management of peritoneal carcinomatosis arising from adenocarcinoma of the colon and rectum. Semin Oncol. 2008;35:183-191

[15] Yan et al. Systematic review on the efficacy of cytoreductive surgery combined with perioperative intraperitoneal chemotherapy for peritoneal carcinomatosis from colorectal carcinoma. J Clin Oncol. 2006;24: 4011-409.

[16] Fajardo et al. Delayed repeated intraperotoneal chemotherapy.. Dis Colon Rectum. Vol 55.: 10 (2012);1044-1052

[17] Soumaoro et al. Coexpression of VEGF-C and Cox-2 in Human Colorectal Cancer and its Association With Lymph Node Metastasis Dis Colon Rectum, March 2006 vol 49 , no 3; 392-398.

[18] Óberg, A. et al. Are lymph node micrometastases of any clinical significance in Dukes stages A and B colorectal cancer? Dis Colon Rectum, October 1998 Vol 41, No. 10 1244-1249.

[19] Edge SB, Byrd DR, Compton CC, et al., eds.: AJCC Cancer Staging Manual. 7th ed. New York, NY: Springer, 2010, pp 143-64.

[20] Wong et al. Staging accuracy in colorectal carcinoma. Journal of Clinical Oncology. Vol 17. no 9 (1999) 2896-2900.

[21] Wang et al. Curative resection of T1 colorectal carcinoma: risk of lymph node metastasis and long-term prognosis. Dis Colon Rectum June 2005. Vol 48, No. 6, 1182-1192.

[22] Kelder et al. Impact of the number of histologically examined lymph nodes on prognosis in colon cancer: a population-based study in the Netherlands. Dis Colon Rectum. Vol. 52 (2009); 260-267.

[23] J. Gastroenterol Heptol 6:325-244. 1991 
[24] Park et al. Comparative analisis of limph node metastasis in patients with ypT0-2 rectal cancers after neoadjuvant chemoradiotherapy. Dis Colon Rectum. Vol 56: 2 (2013).135-141.

[25] Sardon et al. Técnica del ganglio centinela en el cáncer de colon. Experiencia en 125 casos.CIR ESP. 2013; 91(6) 366-371

[26] Watanabe et al. Heterogeneity of KRAS status may explain the subset of discordant KRAS status between primary and metastatic colorectal cancer. Dis Colon Rectum Vol 54: 9(2011) 1170-1178.

[27] Faerden et al. Sentinel node mapping in colonic cancer Dis Colon Rectum. Vol 51: (2008) 891-896

[28] (2013). "Rectal Cancer." National Comprehensive Cancer Network (NCCN) guidelines Retrieved Jun 30, 2013, from www.nccn.org.

[29] Ambiru, S., M. Miyazaki, H. Ito, K. Nakagawa, H. Shimizu, A. Kato, S. Nakamura, H. Omoto and N. Nakajima (1998). "Resection of Hepatic and Pulmonary Metastases in Patients with Colorectal Carcinoma." Cancer 82(2): 274-278.

[30] Carballo, M., M. S. Maish, D. E. Jaroszewski and C. E. Holmes (2009). "Video-Assisted Thoracic Surgery (Vats) as a Safe Alternative for the Resection of Pulmonary Metastases: A Retrospective Cohort Study." J Cardiothorac Surg 4: 13.

[31] Collie, D. A., A. R. Wright, J. R. Williams, B. Hashemi-Malayeri, A. J. Stevenson and C. M. Turnbull (1994). "Comparison of Spiral-Acquisition Computed Tomography and Conventional Computed Tomography in the Assessment of Pulmonary Metastatic Disease." Br J Radiol 67(797): 436-444.

[32] Ehrenhaft, J. L., M. S. Lawrence and D. M. Sensenig (1958). "Pulmonary Resections for Metastatic Lesions." A.M.A. archives of surgery 77(4): 606-612.

[33] Fiorentino, F., I. Hunt, K. Teoh, T. Treasure and M. Utley (2010). "Pulmonary Metastasectomy in Colorectal Cancer: A Systematic Review and Quantitative Synthesis." J $R$ Soc Med 103(2): 60-66.

[34] Gonzalez, M., A. Poncet, C. Combescure, J. Robert, H. B. Ris and P. Gervaz (2013). "Risk Factors for Survival after Lung Metastasectomy in Colorectal Cancer Patients: A Systematic Review and Meta-Analysis." Ann Surg Oncol 20(2): 572-579.

[35] Greelish, J. P. and J. S. Friedberg (2000). "Secondary Pulmonary Malignancy." Surg Clin North Am 80(2): 633-657.

[36] Hellman, S. and R. R. Weichselbaum (1995). "Oligometastases." J Clin Oncol 13(1): 8-10.

[37] 10. Hendriks, J. M., S. Romijn, B. Van Putte, E. Eyskens, J. B. Vermorken, E. Van Marck and P. E. Van Schil (2001). "Long-Term Results of Surgical Resection of Lung Metastases." Acta Chir Belg 101(6): 267-272. 
[38] Inoue, M., Y. Kotake, K. Nakagawa, K. Fujiwara, K. Fukuhara and T. Yasumitsu (2000). "Surgery for Pulmonary Metastases from Colorectal Carcinoma." Ann Thorac Surg 70(2): 380-383.

[39] Irshad, K., F. Ahmad, J. E. Morin and D. S. Mulder (2001). "Pulmonary Metastases from Colorectal Cancer: 25 Years of Experience." Can J Surg 44(3): 217-221.

[40] Jaklitsch, M. T., C. M. Mery, J. M. Lukanich, W. G. Richards, R. Bueno, S. J. Swanson, S. J. Mentzer, B. D. Davis, E. N. Allred and D. J. Sugarbaker (2001). "Sequential Thoracic Metastasectomy Prolongs Survival by Re-Establishing Local Control within the Chest." J Thorac Cardiovasc Surg 121(4): 657-667.

[41] Kondo, H., T. Okumura, Y. Ohde and K. Nakagawa (2005). "Surgical Treatment for Metastatic Malignancies. Pulmonary Metastasis: Indications and Outcomes." Int J Clin Oncol 10(2): 81-85.

[42] Martini, N. and P. M. McCormack (1998). "Evolution of the Surgical Management of Pulmonary Metastases." Chest Surg Clin N Am 8(1): 13-27.

[43] McAfee, M. K., M. S. Allen, V. F. Trastek, D. M. Ilstrup, C. Deschamps and P. C. Pairolero (1992). "Colorectal Lung Metastases: Results of Surgical Excision." Ann Thorac Surg 53(5): 780-785; discussion 785-786.

[44] Mery, C. M., A. N. Pappas, R. Bueno, S. J. Mentzer, J. M. Lukanich, D. J. Sugarbaker and M. T. Jaklitsch (2004). "Relationship between a History of Antecedent Cancer and the Probability of Malignancy for a Solitary Pulmonary Nodule." Chest 125(6): 2175-2181.

[45] Pfannschmidt, J., H. Dienemann and H. Hoffmann (2007). "Surgical Resection of Pulmonary Metastases from Colorectal Cancer: A Systematic Review of Published Series." Ann Thorac Surg 84(1): 324-338.

[46] Pfannschmidt, J., H. Hoffmann and H. Dienemann (2010). "Reported Outcome Factors for Pulmonary Resection in Metastatic Colorectal Cancer." J Thorac Oncol 5(6 Suppl 2): S172-178.

[47] Pfannschmidt, J., T. Muley, H. Hoffmann and H. Dienemann (2003). "Prognostic Factors and Survival after Complete Resection of Pulmonary Metastases from Colorectal Carcinoma: Experiences in 167 Patients." J Thorac Cardiovasc Surg 126(3): 732-739.

[48] Poston, G. J., D. Tait, S. O'Connell, A. Bennett and S. Berendse (2011). "Diagnosis and Management of Colorectal Cancer: Summary of Nice Guidance." BMJ 343: d6751.

[49] Quiros, R. M. and W. J. Scott (2008). "Surgical Treatment of Metastatic Disease to the Lung." Semin Oncol 35(2): 134-146.

[50] Regnard, J. F., D. Grunenwald, L. Spaggiari, P. Girard, D. Elias, M. Ducreux, P. Baldeyrou and P. Levasseur (1998). "Surgical Treatment of Hepatic and Pulmonary 
Metastases from Colorectal Cancers." Ann Thorac Surg 66(1): 214-218; discussion 218-219.

[51] Reinhardt, M. J., N. Wiethoelter, A. Matthies, A. Y. Joe, H. Strunk, U. Jaeger and H. J. Biersack (2006). "Pet Recognition of Pulmonary Metastases on Pet/Ct Imaging: Impact of Attenuation-Corrected and Non-Attenuation-Corrected Pet Images." Eur J Nucl Med Mol Imaging 33(2): 134-139.

[52] Remy-Jardin, M., J. Remy, F. Giraud and C. H. Marquette (1993). "Pulmonary Nodules: Detection with Thick-Section Spiral Ct Versus Conventional Ct." Radiology 187(2): 513-520.

[53] Rena, O., C. Casadio, F. Viano, R. Cristofori, E. Ruffini, P. L. Filosso and G. Maggi (2002). "Pulmonary Resection for Metastases from Colorectal Cancer: Factors Influencing Prognosis. Twenty-Year Experience." Eur J Cardiothorac Surg 21(5): 906-912.

[54] Saisho, S., M. Nakata, S. Sawada, M. Yamashita, H. Saeki, A. Kurita and S. Takashima (2009). "Evaluation of Video-Assisted Thoracoscopic Surgery for Pulmonary Metastases: 11-Years of Experience." Surg Endosc 23(1): 55-61.

[55] Sakamoto, T., N. Tsubota, K. Iwanaga, T. Yuki, H. Matsuoka and M. Yoshimura (2001). "Pulmonary Resection for Metastases from Colorectal Cancer." Chest 119(4): 1069-1072.

[56] Salah, S., K. Watanabe, J. S. Park, A. Addasi, J. W. Park, J. Zabaleta, F. Ardissone, J. Kim, M. Riquet, K. Nojiri, M. Gisabella, S. Y. Kim and K. Tanaka (2013). "Repeated Resection of Colorectal Cancer Pulmonary Oligometastases: Pooled Analysis and Prognostic Assessment." Ann Surg Oncol 20(6): 1955-1961.

[57] Salah, S., K. Watanabe, S. Welter, J. S. Park, J. W. Park, J. Zabaleta, F. Ardissone, J. Kim, M. Riquet, K. Nojiri, M. Gisabella, S. Y. Kim, K. Tanaka and B. Al-Haj Ali (2012). "Colorectal Cancer Pulmonary Oligometastases: Pooled Analysis and Construction of a Clinical Lung Metastasectomy Prognostic Model." Ann Oncol 23(10): 2649-2655.

[58] Villeneuve, P. J. and R. S. Sundaresan (2009). "Surgical Management of Colorectal Lung Metastasis." Clin Colon Rectal Surg 22(4): 233-241.

[59] Weichselbaum, R. R. and S. Hellman (2011). "Oligometastases Revisited." Nature reviews. Clinical oncology 8(6): 378-382.

[60] Yano, T., N. Hara, Y. Ichinose, H. Yokoyama, T. Miura and M. Ohta (1993). "Results of Pulmonary Resection of Metastatic Colorectal Cancer and Its Application." J Thorac Cardiovasc Surg 106(5): 875-879.

[61] Igor T. Gavrilovic and Jerome B. PosnerJournal of Neuro-Oncology (2005) 75: 5-14 Springer 2005 
[62] Tim J. Kruser, Samuel T. Chao,Paul Elson, Gene H. Barnett,Michael A. Vogelbaum, Lilyana Angelov, Robert J. Weil, Robert Pelley, John H. Su CANCER July 1, 2008 / Volume 113 / Number 1

[63] Germán Borobio León, Asunción García Plaza, Roberto González Alconada, Ignacio García Cepeda, Jorge López Olmedo, Alberto Moreno Regidor and David Pescador Hernández A Multidisciplinary Approach to Management, Dr. Giulio A. Santoro

[64] Borobio León, G; García Plaza, A; García Cepeda, I; González Alconada, R.; Hernández Cosido, L. (2010). Metástasis en mano de adenocarcinoma de recto. Un caso excepcional. Cirugía Española 2010;88:195-7.-vol. 88 núm. 03

[65] Bonnheim, D.C; Petrelli, N.J; Herrera, L.; Walsh, D; Mittelman, A.(1986). Osseous metastases from colorectal carcinoma. Am J Surg, vol. 151(4), (April 1986), 457-459.

[66] Chang, H.C; Lew, K.H; Low, C.O .(2001). Metastasis of an adenocarcinoma of the stomach to the 4th metacarpal bone. Hand Surgery 2001 December; 6(2): 239-242.

[67] Clohisy, DR; Perkins, SL; Ramnaraine ML. Review of cellular mechanisms of tumor osteolysis (2000). Clin Orthop 2000;3743:104-114

[68] Desmanet, E; Amrani, M; Fievez, R; Six Ch. Les acrométastases. A propos de deux cas.(1991). Revue de la littérature. Ann Chir Main 1991; 10, n²: 154-157.

[69] Flynn, CJ; Danjoux, C; Wong, J; Christakis, M; Rubenstein, J; Yee, A; et al.(2008). Two cases of acrometastasis to the hands and review of the literature. Curr Oncol 2008 October; 15 (5): 51-58.

[70] Galasko, CS. Diagnosis of skeletal metastases and assessment of response to treatment.(1995). Clin Orthop 1995;312:64-75

[71] García Plaza, A. (2003). Aspectos terapéuticos y pronósticos del carcinoma colorrectal. Ediciones Universidad de Salamanca. (Marzo 2003). Colección Vitor 105.

[72] Healey, J.H; Turnbull, A.D; Miedema, M; Lane, J.M.(1986). Acrometastases. A study of twenty-nine patients with osseous involvement of the hands and feet. .J Bone Joint Surg Am. 1986; 68:743-746.

[73] Kose, F; Sakalli, H ; Sezer, A; Mertsoylu, H; Pourbagher, A; Reyhan, M; Ozyilkan, O. (2008). Colon adenocarcinoma and solitary tibia metastasis: Rare entity. J Gastrointest Canc, vol.39, (February 2008), 146-148.

[74] Méndez López, JM; García Mas, R; Salvà Coll, G. (1997). Metastasis of an adenocarcinoma of the colon to the 1st metacarpal bone. Ann Chir Main Memb Super 1997; 16(2): 134-7.

[75] Mundy, JR \& Yoneda, T. Facilitation and supresion of bone metastasis (1995). Clin Orthop 1995;312:34-44 
[76] Nozue, M; Oshiro, Y; Kurata, M; Seino, K; Koike, N; Kawamoto, T et al (2002). Treatment and prognosis in colorectal cancer patients with bone metastasis. Oncol Rep 2002 Jan-Feb; 9(1): 109-112.

[77] Ross, J.R; Saunders ,Y; Edmonds, P.M; Patel, S; Wonderling, D; Normand, C. (2004). A systematic review of the role of bisphosphonates in metastatic disease. Health Technol Assess. vol 8. (August 2004). 1-176.

[78] Roth, E.S; Fetzer, D.T; Barron,B.J; Usha, A; Joseph, U. A; Isis, W; Gayed, I. W; Wan, D.Q. (2009). Does colon cancer ever metastasize to bone first? a temporal analysis of colorectal cancer progression. BMC Cancer. vol. 9, (August 2009), 274.

[79] Schlüter, K; Gassmann, P; Enns, A.(2006) Organ-Specific Metastatic Tumor Cell Adhesion and Extravasation of Colon Carcinoma Cells with Different Metastatic Potential. The American Journal of Pathology. vol.169, (September 2006),1064-1073.

[80] Schlüter, K; Gassmann, P; Enns, A; Korb, T; Hemping-Bovenkerk, A; Hölzen, J; Haier,J. (2006). Organ-Specific Metastatic Tumor Cell Adhesion and Extravasation of Colon Carcinoma Cells with Different Metastatic Potential. American Journal of Pathology. Vol 169, (September 2006), 1064-1073.

[81] Spiteri, V; Bibra, A; Ashwood, N; Cobb, J. Managing acrometastases treatment strategy with a case illustration (2008). Ann R Coll Surg Engl 2008 October; 90(7): 8-11.

[82] Sundermeyer, M. L; Meropol, N.J; Rogatko, A; Wang, H; Cohen, S.J. (2004). Changing patterns of colorectal cancer metastases: A 10-year retrospective review. Journal of Clinical Oncology, vol 22, no $14 S$,(July 15 Supplement 2004), 3548.

[83] Sundermeyer, M. L; Meropol, N.J; Rogatko, A; Wang, H; Cohen, S.J. (2005).Changing Patterns of Bone and Brain Metastases in Patients with Colorectal Cancer. Clinical Colorrectal Cancer. vol 5, no 2 (July 2005). 108-113. www.intechopen. 